\title{
Ion trapping and cathode bombardment by trapped ions in dc photoguns
}

\author{
E. Pozdeyev* \\ Brookhaven National Laboratory, Upton, New York 11973-5000, USA
}

(Received 19 June 2007; published 6 August 2007)

\begin{abstract}
Ion back bombardment is believed to be a main cause of degradation of quantum efficiency of photocathodes in fixed-voltage photoguns. Ions produced in collisions of the electron beam with residual gas in the cathode-anode gap are accelerated towards the cathode and bombard the cathode surface, reducing its quantum efficiency. In addition to ions produced in the accelerating gap, there is another source of ions that has been overlooked so far in existing models of ion bombardment: ions produced in a beam transport line beyond the anode and trapped in the electron beam. These ions can drift back to the cathode and increase the rate of ion bombardment. This article shows that the flux of trapped ions can significantly exceed the flux of ions produced directly in the accelerating cathode-anode gap. The paper also describes a simple and effective solution to the problem of trapped ions, a positive potential barrier.
\end{abstract}

DOI: 10.1103/PhysRevSTAB.10.083501

PACS numbers: 29.25.Bx, 85.60.Ha

\section{INTRODUCTION}

The successful demonstration of high power energy recovery at JLAB FEL [1] has led to a great interest in developing high average current, high brightness photoinjectors for such applications as energy recovery linac (ERL)-based synchrotron radiation facilities [2,3], FELs [3], electron cooling [4], and colliding beams for nuclear and high energy physics [5]. These applications require beams with an average current of tens to hundreds of milliamps and a normalized emittance of the order of several $\mathrm{mm} \cdot \mathrm{mrads}$ or less. Additionally, nuclear and high energy experiments require a high degree of polarization.

Fixed-voltage (DC) photoemission guns have been successfully used to produce high-quality, high average intensity electron beams for accelerator driven applications [68]. Because of their proven design and ability to produce high average beam current, DC photoguns were selected as a technology of choice for the currently developed Cornell ERL and Daresbury 4GLS [2,3]. While conventional (copper) radio frequency (RF) guns can reach higher accelerating gradients and potentially a lower beam emittance, they have to operate in a pulsed regime to maintain RF power losses under control that limits the average beam current. The superconducting RF (SRF) technology solves the problem of power losses in RF guns without sacrificing the accelerating gradient and is considered as a technology of choice for several future machines [4,5]. However, SRF guns still have to demonstrate reliable long-term operation with a high beam current.

Existing DC photoinjectors have demonstrated a cathode charge lifetime of several hundred Coulombs. Although this extracted charge is adequate for current applications, it becomes exceedingly small for proposed accelerator projects. Cathode bombardment by ions of

\footnotetext{
*pozdeyev@bnl.gov
}

residual gas created in collisions with the electron beam is believed to be a major cause of quantum efficiency $(\mathrm{QE})$ degradation $[6,7,9]$. While exact details of the damage mechanism are not clear, improving vacuum, reducing beam losses, and increasing the laser spot size proved to mitigate the problem of ion back bombardment and increase the cathode charge lifetime.

Existing models of cathode ion bombardment consider only ions produced between the gun cathode and anode. In this paper, we discuss an additional source of ion bombardment that was overlooked so far: ions produced in a beam transport line behind the anode and trapped within the beam. Transversely confined in the beam, these ions can drift towards the cathode-anode gap, accelerate, and damage the cathode surface. As shown in this paper, the flux of trapped ions can significantly exceed the flux of ions produced directly in the cathode-anode gap. In Sec. II of this paper we evaluate stability of trapped ions within the electron beam and estimate the flux of trapped ions. In Sec. III, we introduce a simple solution to the problem of trapped ions, a positive potential barrier, and describe a POISSON model of the new Jefferson Lab load-lock gun with a biased anode. In Sec. IV, we briefly discuss significance of repelling trapped ions in planned high current DC photoguns.

\section{ION TRAPPING}

A typical DC photoemission gun consists of a negatively biased photocathode and a grounded anode separated by a distance of a few centimeters. The cathode is biased to a negative potential of several tens to several hundreds of kilovolts. When the photocathode surface is illuminated with a laser, electrons are emitted from the cathode and accelerated towards the anode, passing through an opening in the anode at the end of acceleration. After that, the beam is transferred through a transport line to other accelerating structures. 
On their path, electrons ionize residual gas, producing low energy ions. Ions produced in the cathode-anode gap are accelerated towards the cathode and damage its surface, reducing QE. Additionally, the beam ionizes residual gas in the transport line behind the anode. These ions are not affected by the accelerating field of the cathode. However, they can get trapped in the electron beam and drift towards the cathode-anode gap. As trapped ions reach the accelerating gap, they get accelerated towards the cathode and damage the cathode surface similarly to ions generated in the gap.

The electric potential induced by a DC electron beam of the radius $a$ is (in the centimeter-gram-second system of units)

$$
\phi= \begin{cases}\frac{2 I}{\beta c}\left(\frac{r^{2}}{2 a^{2}}-\frac{1}{2}+\ln \left(\frac{a}{b}\right)\right) & 0 \leq r \leq a \\ \frac{2 I}{\beta c} \ln \left(\frac{r}{b}\right) & a<r \leq b,\end{cases}
$$

where $b$ is the vacuum pipe radius, $I$ is the beam current, and $\beta c$ is the beam velocity. For a beam pipe radius of $2.5 \mathrm{~cm}$, beam radius of $2.0 \mathrm{~mm}, 100 \mathrm{keV}$ beam energy, and a beam current of $5 \mathrm{~mA}$, Eq. (1) yields a potential well depth of $1.7 \mathrm{~V}$. This is sufficient to trap ions with the average thermal energy, which is approximately $4 \times$ $10^{-2} \mathrm{eV}$ at room temperature. (Note that the energy transferred in the ionization process is mostly absorbed by knocked out electrons.) Equation (1) and the numerical example indicate that ions will be trapped in practically any DC gun if the electron beam current exceeds a few hundred microamps.

Equation (1) does not include the potential of trapped ions, assuming that the beam neutralization degree is small. This condition is easily satisfied for modern DC photoguns with a typical residual gas pressure of the order of $10^{-11}$ Torr. The beam neutralization degree, defined as a ratio of the equilibrium trapped ion density, $n_{i}$, to the electron beam density, $n_{e}$, is equal to the ratio of the lifetime of ions to the ion density buildup time,

$$
\frac{n_{i}}{n_{e}}=\frac{\tau_{l}}{\tau_{t}}
$$

The ion density buildup time is given by

$$
\tau_{t}=\frac{1}{n \sigma \beta c},
$$

where $n$ is the residual gas molecular density and $\sigma$ is the ionization cross section. For hydrogen at $10^{-11}$ Torr, the buildup time is approximately 300 seconds. The lifetime of trapped ions in the beam can be estimated as the time required for an ion to leave the transfer line, drifting along the beam. This time is approximately equal to the length of a free drift between the gun and the first focusing element, $l_{d}$, divided by the ion thermal velocity, $v_{t}$, approximately equal to $1.8 \mathrm{~km} / \mathrm{sec}$ for $\mathrm{H}_{2}$ at room temperature. Assuming the length of the free drift equal to $0.5 \mathrm{~m}$, one obtains the lifetime of ions,

$$
\tau_{l}=\frac{l_{d}}{v_{t}} \approx 3 \times 10^{-4} \mathrm{sec} .
$$

These numbers yield the neutralization degree:

$$
\frac{n_{i}}{n_{e}} \approx 10^{-6},
$$

which is negligible. Note that beam losses and insufficient pumping can lead to an increased level of residual gas pressure in the transfer line. However, gas pressure close to the anode has to reach an unreasonably high value of $10^{-6}$ Torr or so for beam neutralization to affect ion trapping.

DC voltage photoguns are normally operated with a pulsed electron beam produced by a pulsed drive laser. The repetition frequency of electron bunches is a subharmonic of the accelerating RF that accelerates the beam to a higher energy. The potential given by (1) still can be used to analyze ion trapping in a pulsed beam of low intensity. At a high beam current, trapped ions can become unstable in a pulsed beam. The maximum beam current at which transverse ion trapping is still possible can be estimated using the standard matrix analysis that treats interaction of ions with electron bunches as a sequence of thin focusing lenses and drifts. This analysis yields the stability criterion for singly ionized ions of the atomic weight $A$ :

$$
\frac{I}{f_{b}^{2}}<\frac{2 A a^{2} e \beta}{r_{p} c}
$$

where $r_{p}$ is the classical proton radius, $e$ is the elementary charge, $I$ is the average beam current, $f_{b}$ is the bunch repetition rate, and $a$ is the radius of electron bunches. $\mathrm{H}_{2}^{+}$ions will be stable in a pulsed electron beam with an energy of $100 \mathrm{keV}$, bunch radius of $2 \mathrm{~mm}$, and bunch repetition rate of $10 \mathrm{MHz}$ if the beam current is below $0.3 \mathrm{~A}$. The maximum beam current at which trapping of $\mathrm{H}_{2}^{+}$ions is still possible can be obtained for other beam parameters rewriting (6) in the following form:

$$
I=\frac{\beta}{0.55}\left(\frac{a}{2 \mathrm{~mm}}\right)^{2}\left(\frac{f}{10 \mathrm{MHz}}\right)^{2} \cdot 0.3 \mathrm{~A} .
$$

In a typical DC photogun, the beam freely diverges after the anode until it is focused by a solenoid. As the beam size changes along the beam path, the potential on the beam axis also changes according to (1). Equation (1) shows that the longitudinal force experienced by trapped ions is directed towards a smaller beam size, that is, towards the cathode. The portion of trapped ions reaching the cathode depends on the voltage drop produced by the beam along the transfer line and the boundary condition at the focusing solenoid. Assuming that ions reaching the solenoid do not return the cathode-anode gap, one can roughly estimate the portion of trapped ions reaching the cathode as 


$$
\eta \approx \begin{cases}1-\frac{E_{t}}{2 e \delta \phi}=1-\frac{E_{t}}{e \frac{4 l}{\beta c} \ln \left(\frac{a_{f}}{a_{i}}\right)}, & E_{t}<\delta \phi \\ \frac{1}{2}, & \delta \phi<E_{t},\end{cases}
$$

where $E_{t}$ is the average thermal energy of ions, $\delta \phi$ is the total voltage drop one beam axis along the transfer line, $a_{i}$ and $a_{f}$ are the beam radii at the anode and the solenoid, respectively. Thus, the portion of trapped ions reaching the cathode is between $1 / 2$ and unity.

\section{Flux of trapped ions}

The number of ions produced inside the cathode-anode gap and the number of ions produced in the transfer line behind the anode, both normalized to the extracted charge, are given by

$$
\begin{gathered}
\left(\frac{d N}{d Q}\right)_{\text {gap }}=\frac{n}{e} \frac{L_{\text {gap }}}{E_{\max }} \int \sigma(E) d E \\
\left(\frac{d N}{d Q}\right)_{\text {trapped }}=\frac{n}{e} L_{d} \sigma\left(E_{\text {max }}\right),
\end{gathered}
$$

respectively, where $Q$ is the extracted charge, $n$ is the residual gas molecular density, $e$ is the elementary charge, $\sigma(E)$ is the ionization cross section as a function of energy, $E_{\max }$ is the beam energy, $L_{\text {gap }}$ is the length of the accelerating cathode-anode gap, and $L_{d}$ is the length of a free drift after the anode. Figure 1 shows the $\mathrm{H}_{2}$ ionization cross section as a function of the electron beam energy [10].

The new Jefferson Lab load-lock gun, described in detail in [11], accelerates electrons to $100 \mathrm{keV}$. The accelerating cathode-anode gap of the gun, $L_{\text {gap }}$, is equal to 2 inches and the distance to the first dipole corrector, $L_{d}$, is approximately $50 \mathrm{~cm}$. Assuming a residual gas pressure of $5 \times$ $10^{-12}$ and using Eqs. (9) and (10), one can obtain the flux of ions produced in the accelerating gap and the flux of ions

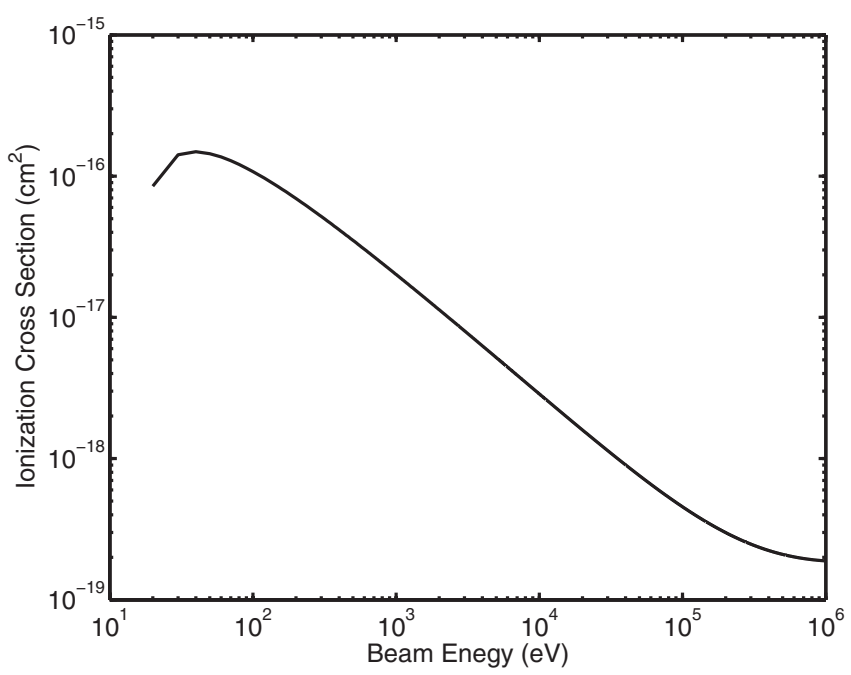

FIG. 1. $\mathrm{H}_{2}$ ionization cross section as a function of the electron beam energy. produced in the transfer line:

$$
\begin{gathered}
\left(\frac{d N}{d Q}\right)_{\text {gap }}=9.7 \times 10^{6} \text { ions } / \mathrm{C} \\
\left(\frac{d N}{d Q}\right)_{\text {trapped }}=2.1 \times 10^{7} \text { ions } / \mathrm{C} .
\end{gathered}
$$

The ratio of (10) to (9) increases with the accelerating voltage. For an accelerating voltage of $650 \mathrm{kV}$ and other parameters used in the last example, the number of ions produced in the accelerating gap and the number of ions produced beyond the anode are, respectively,

$$
\begin{gathered}
\left(\frac{d N}{d Q}\right)_{\text {gap }}=2.4 \times 10^{6} \mathrm{ions} / \mathrm{C} \\
\left(\frac{d N}{d Q}\right)_{\text {trapped }}=1.0 \times 10^{7} \mathrm{ions} / \mathrm{C} .
\end{gathered}
$$

The higher accelerating gradient helps to reduce the flux of ions produced within the accelerating gap. This is because the ionization cross section peaks at low energies. The higher accelerating gradient effectively shortens the volume where the ionization cross section is large and decreases the average cross section.

Damage done by a single ion can depend on the ion energy. Qualitative measurements of QE degradation described in $[6,7,12]$ clearly demonstrated that ions within a wide energy range can effectively damage the cathode surface. However, accurate experimental data on the QE damage efficiency as a function of the ion energy for different cathode types and materials is not readily available. Therefore, this paper does not conclude on how much QE degradation is caused by trapped ions relative to the damage done by ions originating near the cathode.

\section{REPELLING TRAPPED IONS BY A POSITIVE POTENTIAL BARRIER}

An obvious solution to the problem of trapped ions is a positive potential barrier. The optimum location of the potential maximum that minimizes the flux of trapped ions is at the end of the accelerating gap, that is, at the anode. A positive barrier of a few volts should be sufficient to repel trapped ions. Note that the barrier produces some focusing that should be small if the barrier voltage is small comparatively to the accelerating voltage. Also, note that the electrostatic barrier does not change the final beam energy.

\section{Biased anode setup}

To demonstrate the effect of anode biasing on the electrostatic potential in the anode area, a simplified POISSON [13] model of the new JLab load-lock photogun was de- 


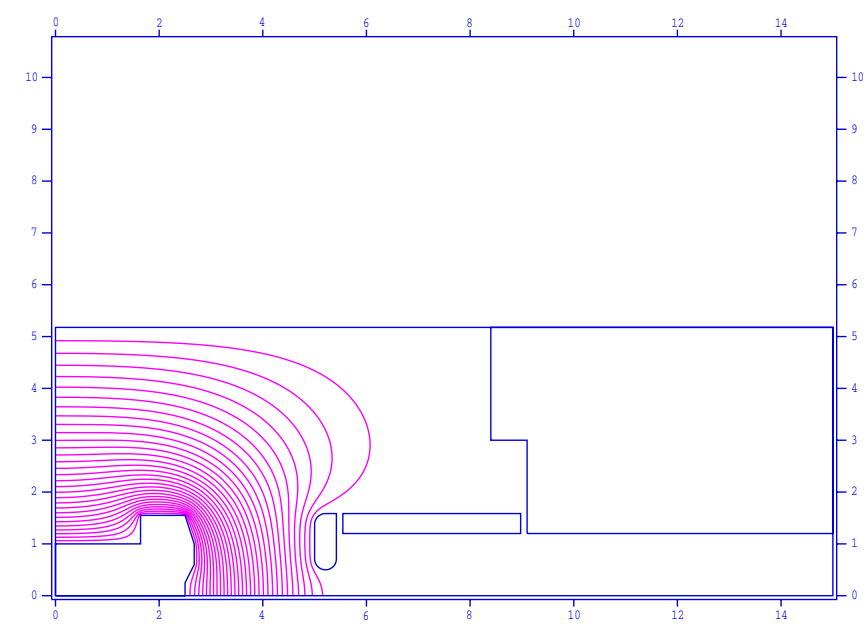

FIG. 2. (Color) Simplified POISSON model of the new JLab loadlock gun cathode region. The horizontal axis is the axis of the gun. The cathode, shown in the lower left corner, is biased to $-100 \mathrm{kV}$. The anode and the anode support are biased to $+2 \mathrm{kV}$ and $+300 \mathrm{~V}$, respectively. The flange supporting the anode structure, shown on the right, is at $0 \mathrm{~V}$.

veloped. The model, shown in Fig. 2, consists of a cathode biased to $-100 \mathrm{kV}$, an isolated anode, an isolated anode support, and a flange, supporting the anode structure. The actual gun operates with the anode and its support at zero potential (grounded). To simulate the potential barrier, the anode in the POISSON model was biased to $+2 \mathrm{kV}$ and the support was biased to $+300 \mathrm{~V}$. Figure 3 shows the electrostatic potential in the anode region produced by the described setup. The created potential barrier is approximately $900 \mathrm{~V}$.

This example shows that the anode has to be biased to a potential noticeably higher than the maximum of the induced potential barrier. This extra voltage is required to compensate the negative potential in the anode area produced by the accelerating field penetrating in the anode opening. The potential droop in the anode area without anode biasing can be clearly observed in Fig. 3. Delivering a biasing voltage of $2 \mathrm{kV}$ to the anode presents no technical problem and can be easily arranged. Photoguns with a lower cathode potential (that is, a higher beam energy) will require higher anode biasing voltage. POISSON simulations for the geometry described above but for a cathode voltage of $-650 \mathrm{kV}$ show that the anode has to be biased to $+5 \mathrm{kV}$ to reproduce a potential barrier of $900 \mathrm{~V}$. This voltage is still reasonably low and can be delivered to the anode with minimal technical difficulties. However, additional requirements such as anode cooling [14] can make anode biasing cumbersome. In cases when anode biasing is undesirable, the potential barrier can be produced by an additional electrode situated behind a grounded anode. Such a setup does not require anode modification and needs less biasing voltage because the electrostatic potential quickly approaches zero behind the grounded anode. In

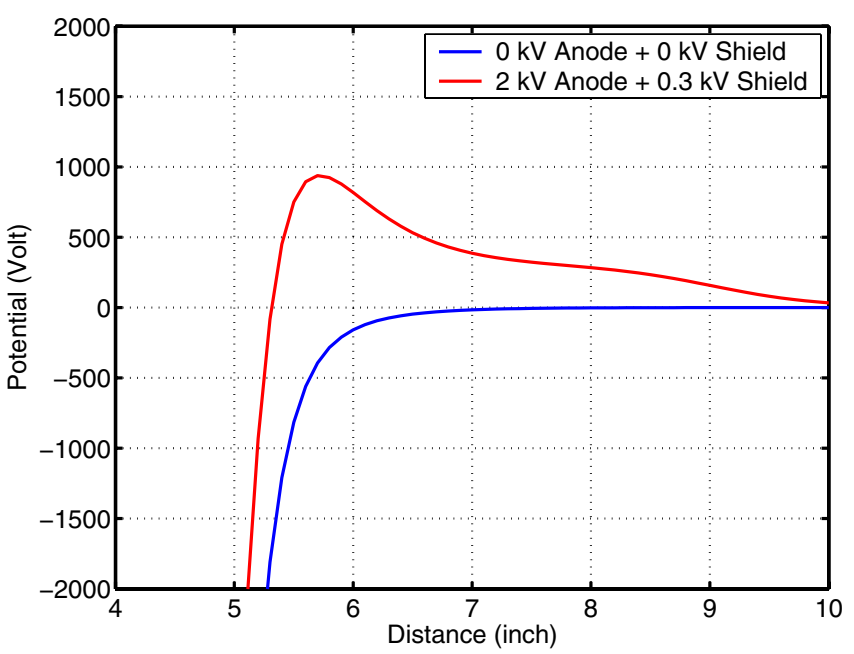

FIG. 3. (Color) Electrostatic potential on the gun axis in the anode area for the actual gun design (blue) and with the biased anode and the anode support (red). The horizontal scale corresponds to that of Fig. 2. The distance 5 inches corresponds to the anode face.

this case, however, the maximum of the potential barrier will be moved farther away from the anode that will let a portion of trapped ions reach the cathode-anode gap. The resulting flux of trapped ions will depend on specifics of a gun design and has to be evaluated for each gun individually.

\section{SIGNIFICANCE FOR FUTURE HIGH CURRENT PHOTOINJECTORS}

Accelerated to the full energy, trapped ions strike the cathode at a location determined by the geometry of the electrostatic field in the cathode-anode gap. Offsetting the laser spot from the area bombarded by trapped ions will mitigate the negative effect of trapped ions on the extracted charge. This method does not require anode biasing or any other gun geometry modification. However, this technique can be used only if the laser spot is sufficiently small and the cathode is sufficiently large. This condition can be hard to satisfy in future high power photoinjectors because of the following reason. Under the assumption that the cathode surface damage rate at the laser spot is proportional to the product of the beam current density and the vacuum pressure, the total extracted charge from a fixed laser spot is proportional to the area of the spot. Thus, a photogun can be operated with a fixed laser spot during a time interval proportional to the spot area divided by the beam current. To keep this interval within a reasonable limit, the area of the laser spot has to be increased at a high beam current. Because future applications require approximately 100 times more average current than that produced by modern DC photoguns, an increase of the laser spot area by more than an order of magnitude is expected. The enlarged laser 
spot size can make clear separation of the laser spot and the area hit by trapped ions impossible in future high intensity DC photoguns. In this case, bombardment by trapped ions can directly limit the cathode charge lifetime. A positive potential barrier on the way of trapped ions provides a simple and effective solution to this problem.

\section{CONCLUSIONS}

It has been demonstrated in this paper that ion trapping in the electron beam can significantly increase cathode ion bombardment in a fixed-voltage photogun. The effect of trapped ions on the cathode lifetime should be more pronounced in guns where the laser spot cannot be clearly offset from the area hit by trapped ions. This can be a concern for future high intensity photoguns where a substantial increase of the laser spot size might be unavoidable in order to extend the cathode lifetime. A positive potential barrier can eliminate the flux of trapped ions to the cathode and is a simple and effective solution to this problem.

\section{ACKNOWLEDGMENTS}

The author would like to thank M. Poelker and J. Grames of Jefferson Lab and K. Aulenbacher of the University of Mainz for their interest in the paper and useful suggestions. Additionally, the author would like to thank M. Poelker for providing a drawing of the new JLab load-lock gun cathode-anode region that was used to create the POISSON model presented in this paper. This work was supported by U.S. DOE under Contract No. DE-AC0298CH1-886.

[1] S. Benson, D. Douglas, P. Evtushenko, K. Jordan, G. Neil, and T. Powers, in Proceedings of LINAC 2006, Knoxville,
Tennessee, 2006, p. 115, available at http://www.jacow.org.

[2] D. H. Bilderback, in Proceedings of the 37th ICFA Beam Dynamics Workshop Future Light Sources (DESY, Hamburg, Germany, 2006), p. 7, available at http:// www.jacow.org.

[3] J.A. Clarke, in Proceedings of EPAC 2006, Edinburgh, Scotland, 2006 (EPS-AG/CERN, Geneva, 2006), p. 181.

[4] I. Ben-Zvi et al., in Proceedings of PAC 2005, Knoxville, Tennessee, 2005 (IEEE, Piscataway, New Jersey, 2005), p. 2741.

[5] V. Litvinenko et al., in Proceedings of PAC 2005, Knoxville, Tennessee, 2005, p. 2768.

[6] K. Aulenbacher et al., Nucl. Instrum. Methods Phys. Res., Sect. A 391, 498 (1997).

[7] C. K. Sinclair, P. A. Adderley, B. M. Dunham, J.C. Hansknecht, P. Hartmann, M. Poelker, J. S. Price, P. M. Rutt, W. J. Schneider, and M. Steigerwald, Phys. Rev. ST Accel. Beams 10, 023501 (2007).

[8] C. Hernandez-Garcia et al., in Proceedings of FEL 2004, Trieste, Italy, 2004 (Comitato Conferenze Elettra, Trieste, 2004), pp. 558-561.

[9] C. K. Sinclair, in Proceedings of PAC 2003, Portland, Oregon, 2003 (IEEE, Piscataway, New Jersey, 2003), p. 76.

[10] M. Reiser, Theory and Design of Charged Particle Beams (John Wiley \& Sons, Inc., New York, 1994).

[11] J. Grames, M. Poelker, P. Adderley, J. Brittian, J. Clark, J. Hansknecht, D. Machie, M. L. Stutzman, and K. SurlesLaw, in Proceedings of SPIN 2006, Kyoto, Japan, 2006 (AIP, Melville, New York, 2007).

[12] K. Aulenbacher et al., in Proceedings of the Workshop on Photocathodes for Polarized Electron Sources for Accelerators, SLAC, Stanford, 1993, p. 1; SLAC-R-432, 1994, http://www.slac.stanford.edu/pubs/slacreports/slacr-432.html.

[13] K. Halbach and R. Holsinger, Part. Accel. 7, 213 (1976).

[14] C. K. Sinclair (private communication). 